\title{
A Case Series of X-Linked Deafness-2 with Sensorineural Hearing Loss, Stapes Fixation, and Perilymphatic Gusher: MR Imaging and Clinical Features of Hypothalamic Malformations
}

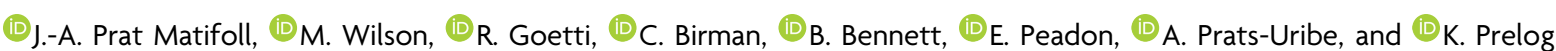

\begin{abstract}
SUMMARY: X-linked deafness-2 (DFNX2) is an X-linked recessive disorder characterized by profound sensorineural hearing loss and a pathognomonic temporal bone deformity. Because hypothalamic malformations associated with DFNX2 have been rarely described, we aimed to further describe these lesions and compare them with features of a nonaffected population. All patients diagnosed with DFNX2 between 2006 and 2019 were included and compared with age-matched patients with normal MR imaging findings and without hypothalamic dysfunction. MR imaging features differing between groups were selected to help identify DFNX2. Sensitivity and specificity were calculated for these features. Agreement among 3 radiologists was quantified using the index $\kappa$. Information on the presence or absence of gelastic seizures, precocious puberty, or delayed puberty was also gathered. We selected distinctive MR imaging features of hypothalamic malformations in DFNX2. The feature selected on axial T2 images was the folded appearance of the ventromedial hypothalamus (sensitivity, 100\%; specificity, 95.8\%) characterized by an abnormal internal/external cleft (sensitivity, 100\%; specificity, $95.7 \%$ ). On coronal T2, the first distinctive feature was a concave morphology of the medial eminence (sensitivity, 100\%; specificity, 97.1\%), the second feature was at least 1 hypothalamic-septum angle $\geq 90^{\circ}$ (sensitivity, 90\%; specificity, $72.5 \%$ ), and the third feature was a forebrain-hypothalamic craniocaudal length of $\geq 6 \mathrm{~mm}$ (sensitivity, 70\%; specificity, 79.7\%). Clinical features were also distinctive because 9 patients with DFNX2 did not present with gelastic seizures or precocious puberty. One patient had delayed puberty. The $\kappa$ index and intraclass correlation coefficient ranged between 0.78 and 0.95 . Imaging and clinical features of the hypothalamus suggest that there is a hypothalamic malformation associated with DFNX2. Early assessment for pubertal delay is proposed.
\end{abstract}

ABBREVIATIONS: CMA = chromosomal microarray; DFNX2 = X-linked deafness-2; ICC = intraclass correlation coefficient; SE = sensitivity; SP = specificity

$\mathbf{X}$ -linked deafness-2 (DFNX2) is an X-linked recessive disorder (Online Mendelian Inheritance in Man No. 304400; https:// www.omim.org/) characterized by profound sensorineural hearing loss with or without a conductive component ${ }^{1}$ and a pathognomonic temporal bone deformity. ${ }^{2,3}$ The pathognomonic inner ear abnormalities include dilation of the inner auditory canal, absence of a lamina cribrosa between the base of the cochlea and the internal auditory canal, ${ }^{4}$ and an absent bony modiolus. ${ }^{5}$ The bony interscalar septa are partially present, and the external dimensions of the cochlea do not differ from normal. ${ }^{6-9}$ The absence of a bone partition

Received December 18, 2019; accepted after revision March 20, 2020.

From the Departments of Radiology (J.-A.P.M., K.P.); Clinical Genetics (M.W.) and Ear Nose Throat (C.B.); Child Development Unit (B.B.); and Deafness Centre (E.P.); Children's Hospital at Westmead (Sydney), Westmead, New South Wales,

Australia; Radiology Department (R.G.), University of Sydney, Children's Hospital at Westmead (Sydney), Westmead, New South Wales, Australia; and Nuffield Department of Orthopaedics, Rheumatology and Musculoskeletal Sciences (A.P.-U.), Centre for Statistics in Medicine, Botnar Research Centre, University of Oxford, Oxford, UK.

Please address correspondence to Joan-Albert Prat Matifoll, MD, The Children's Hospital at Westmead, Radiology Department, Hawkesbury Rd and Hainsworth St, Westmead New South Wales, Australia 2145; e-mail: j.prat@vhebron.net http://dx.doi.org/10.3174/ajnr.A6541 between the inner ear and the internal auditory canal results in a perilymphatic fluid "gusher" during stapes surgery. ${ }^{10,11}$ The disorder is caused by a mutation in the POU domain, class 3, transcription factor 4 (POU3F4) gene (Online Mendelian Inheritance in Man No. 300039) located in chromosome band Xq21. Furthermore, POU3F4 gene malfunction can be caused by inversions and duplications upstream of this gene region. ${ }^{12}$

Because hypothalamic malformations associated with DFNX2 have been rarely described, we aimed to further describe these lesions and compare them with a nonaffected population. ${ }^{13}$

\section{MATERIALS AND METHODS}

This retrospective study included patients with sensorineural hearing loss who underwent MR imaging at a tertiary pediatric hospital (Children's Hospital at Westmead, Sydney) between 2006 and 2019 and demonstrated a pathognomonic DFNX2 inner ear abnormality (Fig 1). A second comparison group of age-matched patients with or without hearing loss and normal MR imaging brain findings was extracted from the same hospital. None of the controls had hypothalamic dysfunction. 


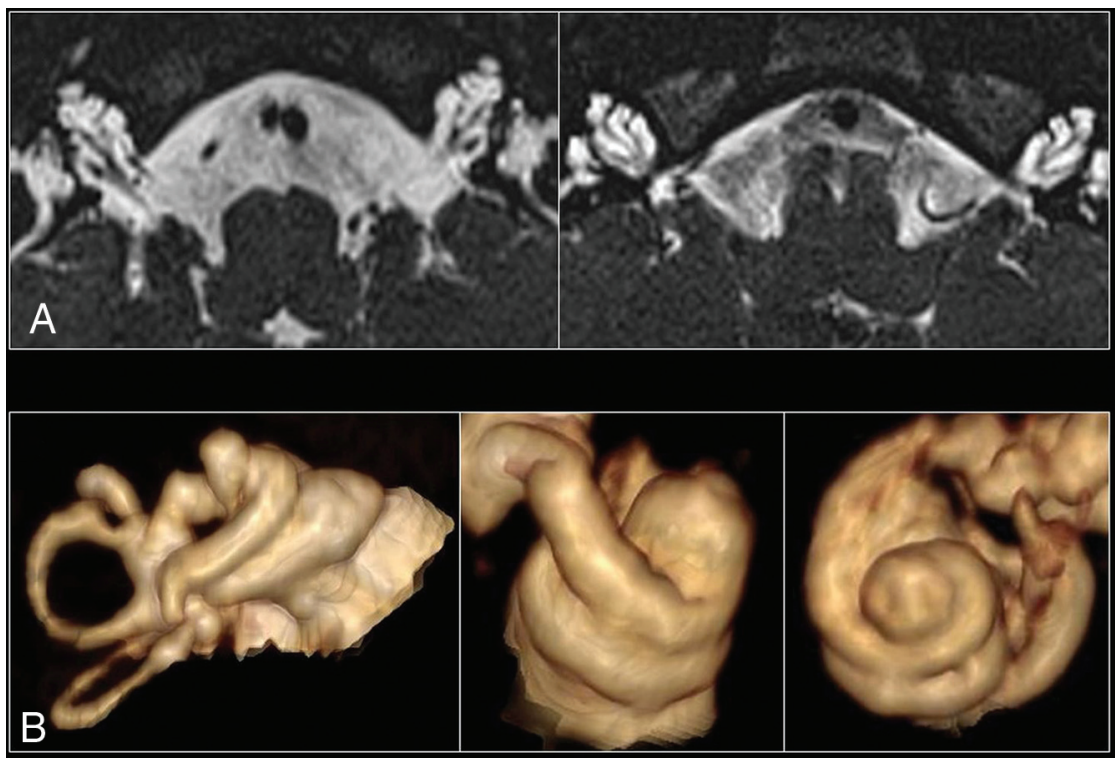

FIG 1. A, High-resolution axial T2 images of both inner ears in a patient with incomplete partition type III. The 3 main features include a dilated internal auditory canal, incomplete separation of the basal turn of the cochlea from the internal auditory canal, with an absent lamina cribrosa and modiolus. $B, 3 D$ reconstructions of several cochleae with incomplete partition type III, which demonstrate the presence of interscalar septa and cochlear turns.

Ethical approval for this study was obtained by the institutional ethics review board (2019/ETH08734).

\section{MR Imaging Protocol}

MR imaging was performed using a 1.5T (Gyroscan Intera and Ingenia; Philips Healthcare) or a $3 \mathrm{~T}$ scanner (Verio; Siemens). While the acquired MR imaging pulse sequences varied during the course of the included studies, at a minimum, the inner ear/auditory canal MR imaging protocol consisted of the following pulse sequences encompassing the inner acoustic canal and inner ear: axial T2 and fast (turbo) spin-echo techniques with special modifications optimizing them for isotropic 3D imaging (T2 sampling perfection with application-optimized contrasts by using different flip angle evolution [SPACE sequence; Siemens]). Other sequences acquired included axial T1 FLAIR, DWI/ADC, gradient-echo, coronal T2/T2 FLAIR, sagittal T1 FLAIR, and sagittal T1 MPRAGE. The matrix size was 320 with voxel size of $0.5 \mathrm{~mm}$, resulting in an FOV of $16 \mathrm{~cm}$ on axial, coronal, and sagittal T2 SPACE sequences.

\section{MR Imaging Analysis}

MR imaging features of the inner ear and hypothalamus were read by 2 pediatric neuroradiologists with 15 and 5 years of experience in pediatric radiology, respectively, and a pediatric radiology fellow; all of them were blinded to the patient group category. We reviewed the following variables:

1) Inner ear: presence of vestibulocochlear and facial nerves and a lamina cribrosa between the basal turn and internal auditory canal; presence of the modiolus and interscalar septa, dysplastic vestibule, and dilated endolymphatic duct.

2) Hypothalamus: axial T2-folded appearance, abnormal cleft side, internal or external cleft, signal intensity on T2WI/ T1WI/T2 FLAIR compared with the orbito-frontal cortex, presence of cystic spaces. Coronal T2: shape of the medial eminence, a low-lying ventromedial hypothalamus in relation to the medial eminence, an angle between the tip of the ventromedial hypothalamus and septum pellucidum, presence of a round lesion, craniocaudal length of the ventromedial hypothalamus tip to the forebrain. Sagittal T2/T1: midline tuber cinereum thickness, infundibular recess morphology, and mammillary body or lamina terminalis involvement.

\section{Genetic Test Data}

Genetic test results from medical records were reviewed by a clinical geneticist. The available genetic tests included chromosomal karyotypes, chromosomal microarrays (CMAs), and single-gene tests (gap junction protein beta 2 [GJB2] gene sequencing \pm gap junction protein beta 6 [GJB6] gene-deletion testing, and POU3F4 gene sequencing).

\section{Clinical Features}

Information about the presence or absence of gelastic seizures, precocious puberty, or delayed puberty at the time of the first MR imaging or during follow-up was gathered.

\section{Statistical Methods}

Quantitative data were described for each group, using mean and SD when normally distributed or median and interquartile range when not. Qualitative data were described using the number of patients and proportions. We compared measurements for the DFNX2 group with the ones in the nonaffected group. We used conditional logistic regression for qualitative measurements and a clustered linear regression for the continuous ones.

We assessed the quality and robustness of our measurements of the inner ear and hypothalamus. For this assessment, we quantified the agreement between observers' measurements and the ones taken by the 2 certified pediatric radiologists and the fellow. We used the intraclass correlation coefficient (ICC) for continuous measures and the $\kappa$ index for qualitative variables. Disagreements between the readers were resolved by consensus. There were no missing data because measures were taken on the available MR imaging scans. No prospective data were analyzed.

\section{RESULTS}

\section{Participants}

There were 11 patients with image-diagnosed DFNX2 between 2006 and 2019 in our population. One patient was 


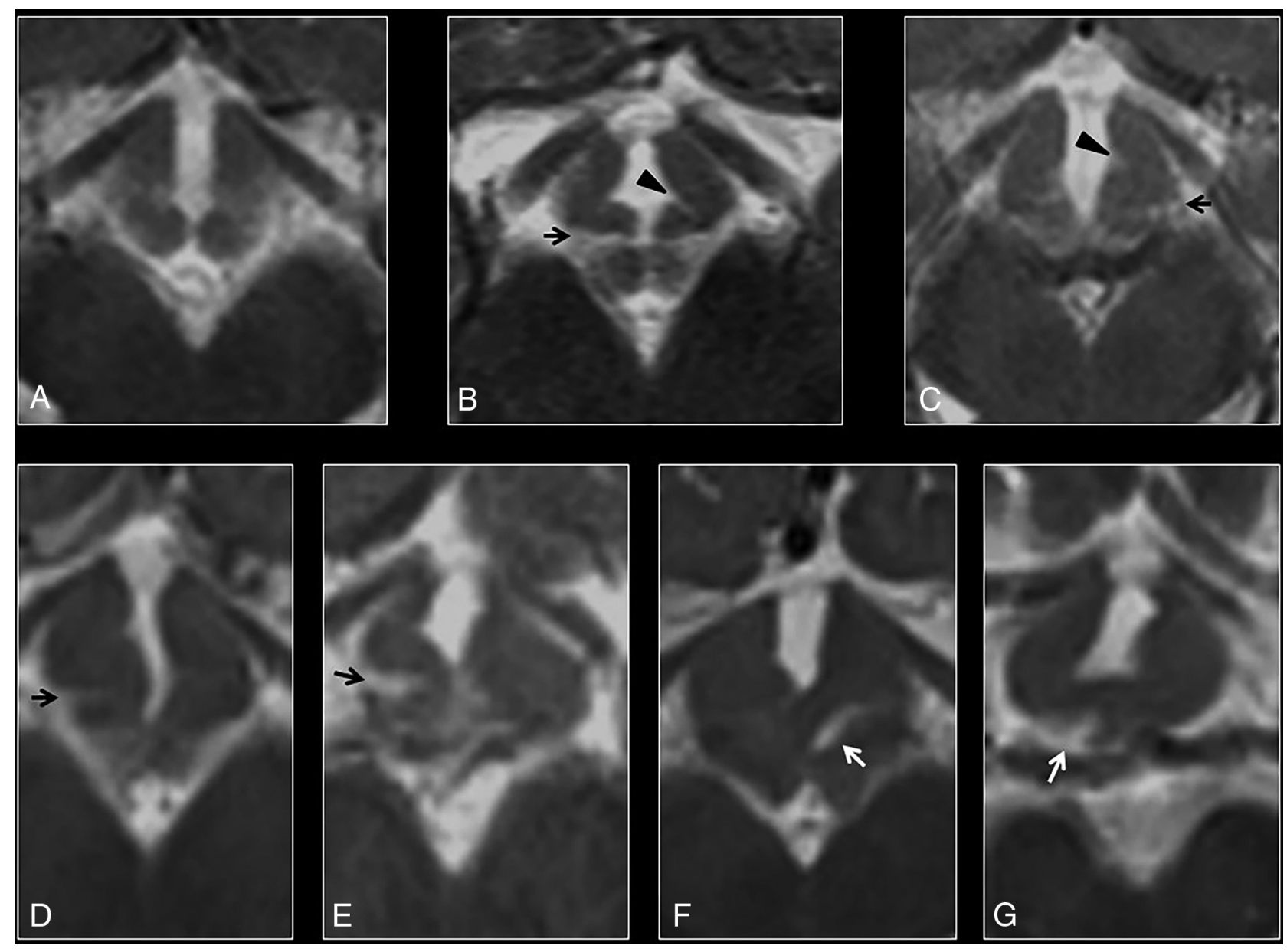

FIG 2. A, Axial T2 image of a normal hypothalamus at the level of the optic radiations. B-G, Axial T2 images of the hypothalamus in a patient with DFNX2, which demonstrate progressive folding of the ventromedial hypothalamus. Note the presence of bilateral clefts in most cases, with external clefts being more easily recognizable (arrows). Internal clefts are also noted on $B$ and C (arrowheads).

excluded due to the lack of MR images available. The remaining 10 patients with DFNX2 were all males and had the pathognomonic temporal bone deformity (Fig 1) with absence of the lamina cribrosa between the basal turn of the cochlea and the internal auditory meatus (IAM) $(\kappa=1)$. Eight patients with DFNX2 had a dysplastic vestibule $(\kappa=0.86)$, and 6 showed a dilated endolymphatic duct $(\kappa=0.61)$. The mean age at the time of performing the first MR imaging was 78.6 months, with a median value of 22 months (range, 6270 months).

The age-matched control group included 69 age-matched patients (younger than 10 years \pm 4 months, 10-18 years \pm 10 months, older than 18 years +28 years). Each patient with DFNX2 younger than 10 years of age had an age-matched control with an age difference of \pm 4 months. Patients with DFNX2 between 10 and 18 years of age had an age-matched control with an age difference of \pm 10 months. The third group of patients older than 18 years of age had age-matched controls with up to 28 years' difference. Furthermore, each patient with DFNX2 had at least 3 age-matched controls with available MR imaging of the internal auditory canal. All patients had normal labyrinth structures.

\section{Hypothalamic Malformations on MR Imaging}

DFNX2 Group versus Age-Matched Control Group

Axial T2 morphology. Morphology of the ventromedial hypothalamus was assessed at the level of the optic radiations. While none of the controls showed hypothalamic overfolding (Fig 2A), 7 patients with DFNX2 demonstrated a folded appearance of the ventromedial hypothalamus $(P<.001)$. This hypothalamic overfolding was characterized by an abnormal external or internal cleft in the ventromedial hypothalamus in 7 patients; most $(n=$ 6) showed bilateral abnormal clefts on axial images rather than unilateral ones (Fig 2B-G). All 7 patients showed external clefts compared with internal clefts (pointing toward the third ventricle), which were only present in 5 patients with DFNX2. None of the controls (67 patients) showed hypothalamic clefts $(P<.001)$, and 2 controls had suboptimal axial images. The $\kappa$ index ranged between 0.76 and 0.95 .

Coronal T2 morphology. Six patients with DFNX2 showed concave morphology of the medial eminence in relation to the pituitary gland, with the lowest point of the ventromedial hypothalamus below the medial eminence $(\kappa=0.78)$. Two patients with DFNX2 showed convex morphology, and 2 had suboptimal images of the hypothalamus on the coronal plane (Fig $3 A-C)$. None of the 


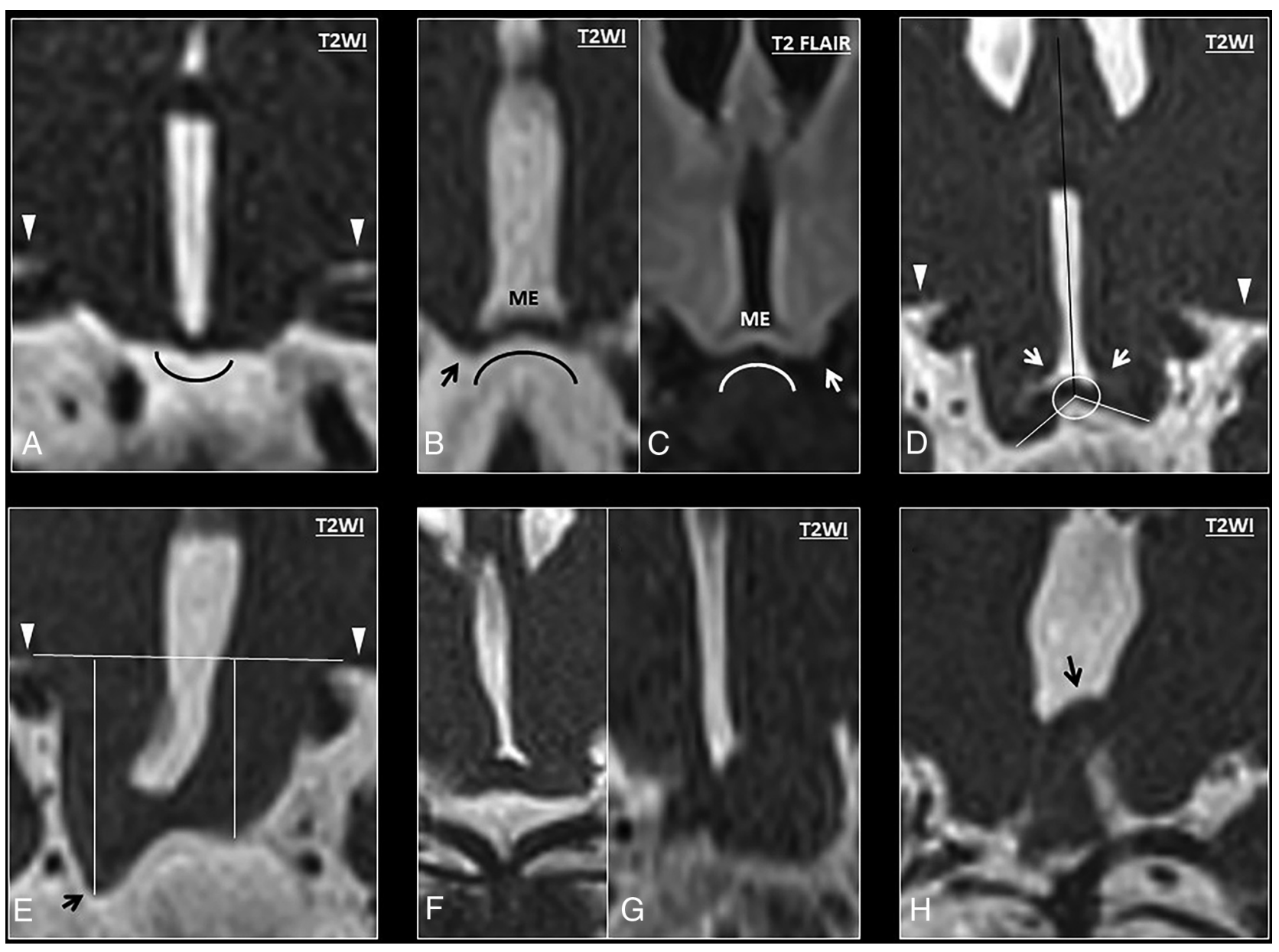

FIG 3. $A$, Coronal $\mathrm{T} 2$ anatomy of a normal hypothalamus showing the classic convex morphology of the medial eminence. $B-H$, High-resolution coronal T2 images show progressive bending of the ventromedial hypothalamus (from mild to severe). B, High-resolution coronal T2 image shows abnormal concavity at the junction between the ventromedial hypothalamus and the medial eminence, with a low-lying ventromedial hypothalamus (black arrow) in relation to the medial eminence (ME). These findings show an abnormal concave morphology of the hypothalamus in relation to the pituitary gland. C, Coronal T2 FLAIR image of another patient with DFNX2, which demonstrates isointense signal compared with the adjacent globus pallidus. Note again the characteristic bending of the ventromedial hypothalamus and its caudal location in relation to the medial eminence (white arrow). D, Measurement of the angle between the tip of the ventromedial hypothalamus and the septum pellucidum (white arrows). E, Measurement of the craniocaudal length of the ventromedial hypothalamus at the lowest point (black arrow) in relation to the basal forebrain (horizontal line). The basal forebrain (white arrowheads) is also indicated on images $A$ and $D$. F and $G$, In some cases, the low-lying hypothalamus and the folding are so severe that some hypothalamic segments appear masslike, though the overall appearance is in keeping with diffuse folding. $H$, Severe hypothalamic bending in a patient with DFNX2, which shows cranial folding of the hypothalamus (black arrow) apart from the typical low-lying or hanging infundibular nucleus (not shown in this image).

controls showed concave medial eminence morphology $(P<.001)$. To further characterize the abnormal hypothalamic concavity on the coronal plane, we measured the coronal hypothalamic-septum pellucidum angle. The first component of this angle was a straight craniocaudal line along the septum pellucidum that crossed the vertex of the concave or convex medial eminence (Fig 3D, black line). The second component was another straight line originating from the previously mentioned vertex of the medial eminence to the most caudal end of the ventromedial hypothalamus; the angle between these 2 lines on either side was named the right and left coronal hypothalamic-septum angle, respectively. The right coronal hypothalamic-septum angle of patients with DFNX2 measured $115.5^{\circ} \pm 17.34^{\circ}$, while it measured $79^{\circ} \pm 8.05^{\circ}$ in age-matched controls $(P<.001)$. The left coronal hypothalamic-septum angle of patients with DFNX2 measured $113.87^{\circ} \pm 16.87^{\circ}$, while it measured $81.87^{\circ} \pm 9.32^{\circ}$ in age-matched controls $(P<.001)$. The individual intraclass correlation coefficient was 0.88 and 0.77 on the right and left sides, respectively.

Another measurement was proposed to further characterize the hypothalamic concavity and low-lying ventromedial hypothalamus: the craniocaudal length between the basal forebrain and the lowest point of the ventromedial hypothalamus. The first component of this measurement was a straight line over the inferior edge of the basal forebrain (Fig 3E, white arrowheads), and the second component was a perpendicular line linking this line with the lowest point of the ventromedial hypothalamus on either side (Fig $3 E$, black arrow). The right forebrain-hypothalamus length in DFNX2 was $7.15 \pm 3.02 \mathrm{~mm}$, while it was $4.26 \pm 0.7 \mathrm{~mm}$ in age-matched controls $(P<.001)$. The left forebrain-hypothalamus length in DFNX2 measured $6.9 \pm 2.1 \mathrm{~mm}$, while it was $4.21 \pm 0.73 \mathrm{~mm}$ in controls $(P<.001)$. The individual intraclass correlation coefficient was 0.92 and 0.87 on the right and left sides, respectively. 


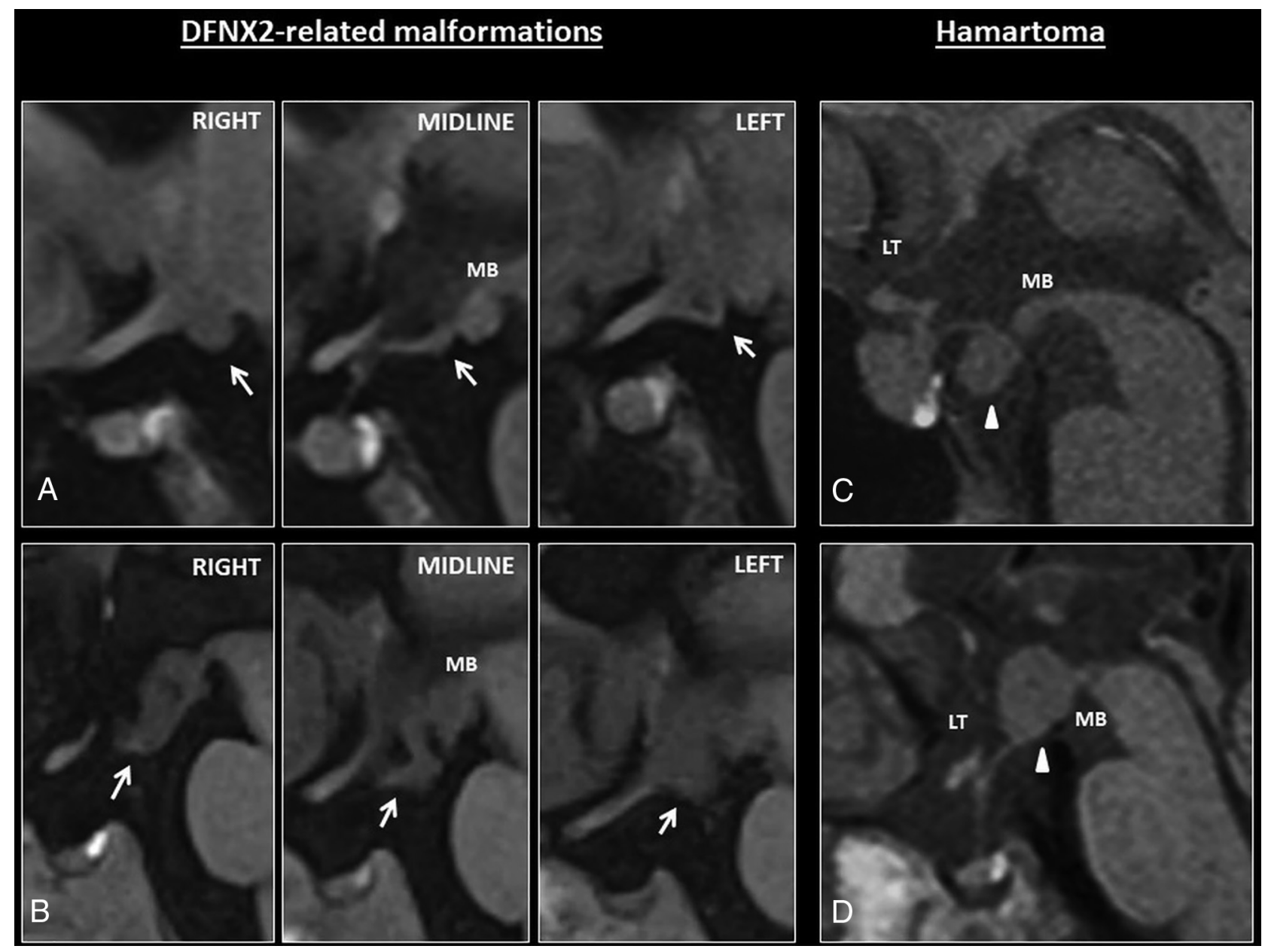

FIG 4. $A$, Sagittal T1 images show mild hypothalamic folding in a patient with DFNX2 (white arrows). This is usually apparent on one of the sagittal slices, such as the left/right one on these series; however, coronal images are better to depict subtle hypothalamic folding. Note the T7-isointense signal of the hypothalamic folding. $B$. The folding is apparent on all the sagittal images through the hypothalamus. Note again the T1isointense signal compared with the adjacent brain parenchyma. $C-D$, Hypothalamic hamartomas tend to be masslike rather than cause hypothalamic folding. They usually arise from the tuber cinereum protruding caudally toward the suprasellar cistern or grow within the third ventricle (white arrowheads) and tend to involve adjacent structures such as the mamillary bodies. MB indicates mamillary bodies; LT, lamina terminalis.

Sagittal T1/T2 morphology. None of DFNX2 hypothalamic malformations showed infundibular, mammillary body, or lamina terminalis involvement (Fig $4 A,-B$ ).

Signal intensity. The hypothalamic overfolding was isointense on T2 FLAIR in all patients with available T2 FLAIR sequences $(P=.44)$, isointense on T2 compared with the adjacent orbitofrontal cortex in all cases, and isointense on T1. The $\kappa$ index ranged between 0.72 and 0.82 . Only 3 patients had available magnetic susceptibility sequences, none showed blooming artifacts, and only 1 was administered contrast, without identifying contrast enhancement.

Incidental findings in patients with DFNX2: Two patients with DFNX2 had a retrocerebellar cyst, 2 had a Meckel cave cyst, 2 had temporal arachnoid cysts, another patient had a cavum septum vergae, and 1 had a cavum velum interpositum.

\section{Proposed MR Imaging Features of Hypothalamic Malformation in DFNX2}

On axial T2 images. The folded appearance of the ventromedial hypothalamus (sensitivity [SE], 100\%; specificity [SP], 95.8\%) characterized by an abnormal internal/external cleft (SE 100\% SP 95.7\%) showed high specificity and sensitivity as well as interobserver agreement for patients with DFNX2 (Fig 2).

On coronal T2 images. A concave morphology of the medial eminence in relation to the pituitary gland with the lowest point of the ventromedial hypothalamus below the medial eminence (Fig 3) also showed high specificity and sensitivity (SE, 100\%; SP, 97.1\%). Furthermore, 2 measurements could help distinguish DFNX2 hypothalamic malformations from features in healthy patients: at least 1 hypothalamic-septum angle of $\geq 90^{\circ}$ (SE, 90\%; SP, $72.5 \%)$ as well as 1 forebrain-hypothalamic craniocaudal length of $\geq 6 \mathrm{~mm}$ (SE 70\%; SP 79.7\%). The presence of at least 3 abnormal variables was sufficient to rule out a normal hypothalamus (Table).

\section{Clinical Results}

There was no clinical history of gelastic seizures or precocious puberty in 9 patients with DFNX2. One patient had delayed puberty, and no clinical information was available for 1 patient. 


Axial T2
Folded appearance
Bilateral abnormal internal or external cleft
Coronal T2
Concave medial eminence
Right hypothalamic-septum angle
Left hypothalamic-septum angle
Right forebrain-hypothalamus length (mm)
Left forebrain-hypothalamus length (mm)

Note: $-\kappa$ indicates the Cohen $\kappa$ coefficient.

${ }^{a}$ Data are mean values \pm standard deviation. $P$ values correspond to Wilcoxon/Mann-Withney test for differences in means of DFNX2 versus age-matched controls.
Genetic Test Results. Eight of 10 patients with DFNX2 had some genetic testing recorded. Two patients (brothers) had a POU3F4 gene mutation (X:82763940:AAAG $>$ A, NM_000307.4:c.614_ 616delGAA, p.Arg205del) detected by targeted sequencing. One also had a CMA-detected duplication variant of unknown significance in $14 \mathrm{q} 24.3$, considered clinically insignificant. Two other patients (brothers) had a CMA-detected Xq21.1 deletion $\sim 600$ to $\sim 1700$ Kilobase $(\mathrm{kb})$ upstream of POU3F4, considered causative because deletions located $\sim 10$ to $\sim 970 \mathrm{~kb}$ upstream of POU3F4 are reported in some patients with DFNX2, suggesting that they contain regulatory elements of the structural gene itself. ${ }^{14}$ All 4 patients had normal sequencing of GJB2. Four additional patients had GJB2 sequencing \pm GJB6 deletion testing, with normal findings. One of a pair of brothers had a CMA-detected 1q21.11q21.2a duplication variant, involving a known neurodevelopmental susceptibility locus, not known to be associated with hearing loss; this was not present in his brother. The 2 others did not have CMA testing, but 1 had a normal karyotype. The last 2 patients had not undergone any genetic testing. Thus, of the 6 patients not yet confirmed by genetic testing, 4 have not yet had CMA testing and none have had POU3F4 sequencing. The lack of genetic confirmation was a limitation in our study, though all patients without genetic confirmation presented with the pathognomonic temporal bone deformity described in DFNX2.

\section{DISCUSSION}

Hypothalamic malformations in our patients with DFNX2 demonstrate the aforementioned folded appearance on axial images and concave morphology on coronal images without signal abnormalities. To date, there are 2 case reports and 1 case series in the literature describing hypothalamic malformations or hamartomatouslike lesions in patients with DFNX2. Whitehead et $\mathrm{al}^{15}$ described a ventrolateral tuber cinereum diverticulum associated with other midline abnormalities in a patient with $\mathrm{Xq} 21$ deletion involving the POU3F4 gene. Anderson et $\mathrm{al}^{16}$ described a 4 -year-old boy with sensorineural hearing loss, autism, and a hypothalamic mass just anterior to the mammillary bodies that was isointense on both T1and T2-weighted sequences, without restricted diffusion, susceptibility artifacts, or enhancement, which was labeled as an asymptomatic hypothalamic hamartoma. No hormonal disturbance was identified before 10 years of age. Whole-exome sequencing revealed a mutation of the POU3F4 gene (p.G216E, hemizygous). Siddiqui et $\mathrm{al}^{17}$ reported a series of 12 patients with DFNX2 and mild-tosevere but characteristic dysmorphism of the hypothalamus. These malformations ranged from a thickened and irregular hypothalamus to hamartoma-like hypothalamic enlargement. They were isointense to gray matter on T1WI and iso- or slightly hyperintense on T2WI and showed no contrast enhancement. None of the patients had seizures or endocrinologic abnormalities.

The possible mechanism linking the pathognomonic inner ear deformity and hypothalamic malformations was described by Andersen and Rosenfeld, ${ }^{18}$ who stated that a DNA transcription factor involved in the development of the nervous system, hypothalamus, pituitary gland, and inner ears was encoded by POU3F4. This gene also plays a role in the formation of hypothalamic nuclei and regulation of the proglucagon promoter.

Our case series is the second largest available and the largest case series from a single institution. Our clinical results confirm the association described by Siddiqui et $\mathrm{al}^{17}$ between DFNX2 and hypothalamic malformations. Moreover, we pinpoint specific MR imaging features and measurements that could help further describe these malformations to identify subtle malformations that could otherwise be overlooked. Our proposed features and measurements to describe DFNX2-related hypothalamic malformations are as follows: On axial T2 images, the folded appearance of the ventromedial hypothalamus is characterized by an abnormal cleft (predominantly external and bilateral). On coronal T2 images, the first distinctive feature would be a concave morphology of the medial eminence in relation to the pituitary gland, the second feature would include at least 1 hypothalamic-septum angle above $90^{\circ}$, and the third feature would consist of at least 1 forebrain-hypothalamic craniocaudal length above $6 \mathrm{~mm}$. On sagittal T1 images, the lack of infundibular, mamillary body, or lamina terminalis involvement could be helpful because it was not identified in any DFNX2 case. Furthermore, our population of patients with DFNX2 also appeared to show variable cystic dilations of subarachnoid spaces (retrocerebellar cysts, Meckel cave cysts, and temporal arachnoid cysts). The presence of these cystic dilations might be associated with a broad spectrum of hypothalamic anomalies because 
arachnoid cysts have also been described in patients with hypothalamic hamartoma by Booth et al. ${ }^{19}$

Clinically, none of patients with DFNX2 with available clinical information $(n=9)$ presented with gelastic seizures or precocious puberty, while patients with hypothalamic hamartomas usually present with gelastic seizures, precocious puberty, or both. ${ }^{20}$ One of our cohort had delayed puberty without any evidence of hormonal disturbance, and his brother was lost to follow-up. The remaining patients are prepubescent; therefore, clinical follow-up to assess for pubertal delay may be helpful.

\section{Limitations}

There were 3 main limitations to our study: First, the small number of patients affected by this rare pathology; and second, the presence of 3 pairs of brothers in our sample. Third, although all had the DFNX2 clinical phenotype, only 4 of our patients (2 pairs of brothers) have had confirmatory genetic testing. Of the other 6 patients, 4 have not yet had a CMA and none have had POU3F4 sequencing.

\section{CONCLUSIONS}

MR imaging and clinical features of the hypothalamus in patients with DFNX2 suggest that there is a hypothalamic malformation associated with DFNX2 that does not behave clinically like a typical hypothalamic hamartoma. We expect our research to help differentiate DFNX2-related hypothalamic malformations and their clinical behavior from a normal hypothalamus and classic hypothalamic hamartoma because DFNX2-related hypothalamic malformations may be misinterpreted as a neoplasm or hamartoma and therefore lead to unnecessary further investigations. Early assessment for pubertal delay is proposed.

Disclosures: Catherine Birman—UNRELATED: Board Membership: The Royal Institute for Deaf and Blind Children (RIDBC), Comments: only on an unpaid board; Payment for Lectures Including Service on Speakers Bureaus: lectures for Cochlear Investments Pty Ltd; Travel/Accommodations/Meeting Expenses Unrelated to Activities Listed: for some conferences supported by Cochlear Investments Pty Ltd or MED-EL, Comments: support for attendance at some conferences such as accommodations or registration or travel if an invited speaker.* *Money paid to the institution.

\section{REFERENCES}

1. Bitner-Glindzicz M, Turnpenny P, Höglund P, et al. Further mutations in Brain 4 (pou3f4) clarify the phenotype in the $\mathrm{x}$-linked deafness, dfn3. Hum Mol Genet 1995;4:1467-69 CrossRef Medline

2. Nance WE, Setleff R, McLeod A, et al. X-linked mixed deafness with congenital fixation of the stapedial footplate and perilymphatic gusher. Birth Defects Orig Artic Ser 1971;07:64-69 Medline
3. Sennaroğlu L, Bajin MD. Incomplete partition type III: a rare and difficult cochlear implant surgical indication. Auris Nasus Larynx 2018;45:26-32 CrossRef Medline

4. Phelps PD, Reardon W, Pembrey M, et al. X-linked deafness, stapes gushers and a distinctive defect of the inner ear. Neuroradiology 1991;33:326-30 CrossRef Medline

5. Talbot JM, Wilson DF. Computed tomographic diagnosis of $\mathbf{X}$ linked congenital mixed deafness, fixation of the stapedial footplate, and perilymphatic gusher. Am J Otol 1994;15:177-82 Medline

6. Huang BY, Zdanski C, Castillo M. Pediatric sensorineural hearing loss, Part 1: practical aspects for neuroradiologists. AJNR Am J Neuroradiol 2012;33:211-17 CrossRef Medline

7. Sennaroglu L, Saatci I. A new classification for cochleovestibular malformations. Laryngoscope 2002;112:2230-41 CrossRef Medline

8. Huang BY, Zdanski C, Castillo M. Pediatric sensorineural hearing loss, Part 2: syndromic and acquired causes. AJNR Am J Neuroradiol 2012;33:399-406 CrossRef Medline

9. Özbal Batuk M, Çınar BÇ, Özgen B, et al. Audiological and radiological characteristics in incomplete partition malformations. Int $A d v$ Otol 2017;13:233-38 CrossRef Medline

10. Pembrey M, Ropers H, Monaco A, et al. Association between Xlinked mixed deafness and mutations in the POU domain gene POU3F4. Science 1995;267:685-88 CrossRef Medline

11. Lee H, Choi J, Kim S, et al. Clinical evaluation of DFN3 patients with deletions in the POU3F4 locus and detection of carrier female using MLPA. Clin Genet 2010;78:524-32 CrossRef Medline

12. Haaf T, Hofrichter MA, Vona B, et al. Non-syndromic hearing loss gene identification: A brief history and glimpse into the future. Mol Cell Probes 2015;29:260-70 CrossRef Medline

13. Baroncini M, Jissendi $P$, Balland E, et al. MRI atlas of the human hypothalamus. Neuroimage 2012;59:168-80 CrossRef Medline

14. Naranjo S, Voesenek K, de la Calle-Mustienes E, et al. Multiple enhancers located in a 1-Mb region upstream of POU3F4 promote expression during inner ear development and may be required for hearing. Hum Genet 2010;128:411-19 CrossRef Medline

15. Whitehead MT, Vezina G. Tuber cinereum diverticula in a 28-monthold with Xq21 deletion syndrome. Case Rep Radiol 2014;2014:1-4 CrossRef

16. Anderson EA, Özütemiz C, Miller BS, et al. Hypothalamic hamartomas and inner ear diverticula with $\mathrm{X}$-linked stapes gusher syndrome: new associations? Pediatr Radiol 2020;50:142-45 CrossRef Medline

17. Siddiqui A, D'Amico A, Colafati GS, et al. Hypothalamic malformations in patients with $\mathrm{X}$-linked deafness and incomplete partition type 3. Neuroradiology 2019;61:949-52 CrossRef Medline

18. Andersen B, Rosenfeld MG. POU domain factors in the neuroendocrine system: lessons from developmental biology provide insights into human disease. Endocr Rev 2001;22:2-35 CrossRef Medline

19. Booth TN, Timmons C, Shapiro K, et al. Pre- and postnatal MR imaging of hypothalamic hamartomas associated with arachnoid cysts. AJNR Am J Neuroradiol 2004;25:1283-85 Medline

20. Harrison VS, Oatman O, Kerrigan JF. Hypothalamic hamartoma with epilepsy: review of endocrine comorbidity. Epilepsia 2017;58: (Suppl 2):50-59 CrossRef 